\title{
Scaling laws for diamond chemical-vapor deposition. II. Atomic hydrogen transport
}

\author{
D. G. Goodwin \\ Division of Engineering and Applied Science, California Institute of Technology, Pasadena, \\ California 91125
}

(Received 14 June 1993; accepted for publication 31 July 1993)

\begin{abstract}
Scaling relations are developed to allow estimation of the atomic hydrogen concentration at the substrate during diamond chemical-vapor deposition for both diffusion-dominated and convection-dominated reactors. In the convection-dominated case, it is shown that there exists an optimal Mach number which maximizes the $\mathrm{H}$ concentration delivered to the substrate. In addition, when homogeneous recombination is taken into account, there exists an optimal operating pressure. This analysis shows that a sonic flow of highly dissociated hydrogen at a pressure near atmospheric is optimal for rapid growth of high-quality diamond.
\end{abstract}

\section{INTRODUCTION}

To scale up diamond chemical-vapor deposition processes, two factors must be considered. The dependence of film growth rate and quality on the local chemical environment at the substrate must be known, and the relation of this environment to controllable process parameters must also be understood. In a companion paper to this one ${ }^{1}$ we have presented a simplified model of diamond surface chemistry, which results in simple approximate expressions for the growth rate and defect density in terms of the concentrations of $\mathrm{H}$ and $\mathrm{CH}_{3}$ at the substrate. It was shown that a simple defect formation model, in which defects are assumed to result from interaction of surface species, leads to a relationship between the defect density $X_{\text {def }}$, the linear growth rate $G$, and the atomic hydrogen concentration at the surface $[\mathrm{H}]_{0}$,

$$
X_{\mathrm{def}} \propto \frac{G}{\left([\mathrm{H}]_{0}\right)^{n}},
$$

where $n \approx 2$. This expression shows that increasing the growth rate (for example, by adding additional hydrocarbon) requires a compensating increase in the atomic hydrogen concentration to maintain the same film quality. This qualitative conclusion is in accord with much experience in diamond film growth, and with a similar analysis by Butler and Woodin. ${ }^{2}$

Therefore, a critical issue for process scale-up and optimization is to determine the scaling of $[\mathrm{H}]_{0}$ with process parameters. This question is complex, since the $\mathbf{H}$ concentration at the substrate is determined by a balance between surface recombination and transport to the surface, either by diffusion or convection, and may be affected by homogeneous recombination as well.

In this article the question of $\mathrm{H}$ transport under diamond chemical-vapor-deposition (CVD) conditions is examined in detail. Simple scaling relations are derived for the effects of pressure, flow velocity, substrate diameter, and degree of gas activation on the $H$ concentration at the substrate, for both diffusion-dominated and convectiondominated reactors.
After first deriving the basic relations valid for all reactors, transport in low-pressure diffusion-dominated reactors is considered, followed by a detailed discussion of $\mathrm{H}$ transport in high-growth-rate, convection-dominated reactors (primarily plasma and combustion torches). In the latter case, it is shown that there exists an optimal Mach number and pressure for $\mathbf{H}$ transport. Expressions are also given for the heat flux to the substrate.

For most of the results presented here, simple transport theory is used to derive the scaling relations. In some cases, particularly when gas-phase chemistry is considered, these results are supplemented by numerical simulations of the flow. In this way we may assess the importance of effects (such as variable transport properties, large temperature gradients, and gas-phase chemistry) which we neglect in the simple theoretical derivations.

The code used for the simulations is one we have developed and used extensively for diamond CVD modeling. ${ }^{3-7}$ In this code, the governing transport equations for axisymmetric flow are solved with appropriate boundary conditions to determine the velocity, temperature, and concentration profiles near the surface. Both heterogeneous and homogeneous chemistry are included, along with variable transport properties calculated using the subroutines of Kee et al. ${ }^{8}$ Green et $a L^{7}$ have recently compared species and temperature profiles in the stagnation-point boundary layer of an atmosphericpressure, inductively coupled plasma torch calculated using this code with measured profiles obtained with degenerate four-wave mixing, and find good agreement.

The general equations derived here are evaluated numerically for the case of a dilute hydrocarbon in hydrogen gas mixture. These evaluated formulas are given in mixed units, with lengths in $\mathrm{cm}$ and the pressure in Torr. The substrate temperature is assumed to be $1200 \mathrm{~K}$ in all cases.

\section{BASIC EQUATIONS}

For the purposes of species transport to the surface, the Peclet number provides a useful means of classifying different diamond growth methods. ${ }^{9}$ The Peclet number is defined by $\mathrm{Pe}=u L / D$, where $u$ is a characteristic flow 
velocity, $L$ a characteristic length scale (for example, the substrate diameter), and $D$ is the diffusion coefficient for the species in question. If $P e<1$, species transport is dominated by diffusion, and is independent of the gas velocity. Low-pressure, low-velocity diamond CVD reactors (for example, conventional hot-filament and microwave systems) operate in this regime. ${ }^{3,9-11}$

In the opposite limit, $\mathrm{Pe}>1$, convective species transport is dominant. High-growth-rate methods (combustion torches, plasma jets) typically operate in this regime. In this case, transport of species in regions far from any surface is primarily due to bulk fluid motion. However, even if $P e>1$ there is always a thin fluid layer near all solid surfaces where diffusive transport dominates, since the velocity must go to zero at the surface. This thin layer, through which species must diffuse to reach the surface, is the boundary layer. The characteristics of the boundary layer determine $\mathrm{H}$ transport to the substrate in these systems.

A parameter similar to the Peclet number is the Reynolds number, defined by $\operatorname{Re}=u L / v$, where $v$ is the kinematic viscosity of the gas. The Reynolds number determines the nature of the velocity field, and governs the transition of turbulence. For gases, $\mathrm{Re}$ and $\mathrm{Pe}$ are similar in magnitude, since the ratio $v / D$ (the Schmidt number) is of order one. For example, for $\mathrm{H}_{2}$ diffusing through air, $v / D=0.22$, while for $\mathrm{CO}_{2}$ diffusing through $\mathrm{H}_{2}$ $v / D=1.58 .^{12}$ A third transport property important for heat transfer is the thermal diffusivity $\alpha$. This, too, is similar in magnitude to $v$ for most gases. For most diatomic gases at reasonable temperatures, the ratio $v / \alpha$ (the Prandtl number) is approximately 0.7 .

Since the gas velocity is zero at the surface whatever the value of $\mathrm{Pe}$ (neglecting the small Stefan velocity due to net mass deposition), the molar flux of $\mathrm{H}$ to the surface $j_{\mathrm{H}}$ may be expressed as ${ }^{13}$

$$
j_{\mathrm{H}}=n_{0} D_{\mathrm{H}}\left(\frac{d X_{\mathrm{H}}}{d z}\right)_{z=0},
$$

where $n_{0}=p / R T_{0}$ is the molar gas density at the surface, $D_{\mathrm{H}}$ is the diffusion coefficient for atomic hydrogen, $X_{\mathrm{H}}$ is the atomic hydrogen mole fraction, and $z$ is the distance from the surface.

At steady state, the flux of $\mathbf{H}$ to the surface will just balance the loss of $\mathbf{H}$ due to surface recombination, and therefore

$$
j_{\mathrm{H}}=\dot{R}_{\mathrm{H}},
$$

where $\dot{R}_{\mathrm{H}}$ is the surface recombination rate, given by

$$
\dot{R}_{\mathrm{H}}=\gamma_{\mathrm{H}} \frac{[\mathrm{H}] \bar{c}_{\mathrm{H}}}{4} .
$$

Here $\gamma_{\mathrm{H}}$ is the recombination coefficient, and $\bar{c}_{\mathrm{H}}$ is the mean thermal speed of a $\mathbf{H}$ atom.

Recently, two groups have reported measurements of $\gamma_{\mathrm{H}}$ at temperatures near $1200 \mathrm{~K} .^{14,15}$ Both studies indicate $\gamma_{\mathrm{H}}$ at this temperature is slightly greater than 0.1 , indicating that $H$ recombines readily on the diamond surface.

Substituting from Eqs. (2) and (4) into Eq. (3), the $\mathrm{H}$ mole fraction at the surface $X_{\mathrm{H}, 0}$ may be expressed as

$$
X_{\mathrm{H}, 0}=\frac{4 D_{\mathrm{H}}}{\gamma_{\mathrm{H}} \overline{\mathcal{C}}_{\mathrm{H}}}\left(\frac{d X_{\mathrm{H}}}{d z}\right)_{z=0} .
$$

The $\mathrm{H}$ concentration at the surface is then given by

$$
[\mathbf{H}]_{0}=n_{0} X_{\mathbf{H}, 0} .
$$

Let us define a characteristic diffusion length scale $l_{d}$ such that

$$
\left(\frac{d X_{\mathrm{H}}}{d z}\right)_{z=0}=\frac{X_{\mathrm{X}, \mathrm{ref}}-X_{\mathrm{H}, 0}}{l_{d}},
$$

where $X_{\mathrm{H}, \mathrm{ref}}$ is the $\mathrm{H}$ mole fraction at some specified "reference" position (for example, the filament surface in a hot-filament reactor, or a point outside the boundary layer in a high-Pe reactor). Then Eq. (2) may be rewritten as

$$
j_{\mathrm{H}}=\frac{n_{0} D_{\mathrm{H}}}{l_{d}}\left(X_{\mathrm{H}, \mathrm{ref}}-X_{\mathrm{H}, 0}\right),
$$

and Eq. (5) as

$$
\frac{X_{\mathrm{H}, 0}}{X_{\mathrm{H}, \mathrm{ref}}}=\left(1+\frac{\gamma_{\mathrm{H}} l_{d} \bar{c}_{\mathrm{H}}}{4 D_{\mathrm{H}}}\right)^{-1} \text {. }
$$

The quantity $l_{d}$ is clearly an important parameter for $\mathrm{H}$ transport: $\mathrm{H}$ at the surface will be maximized when $l_{d}$ is made as small as possible. (In the mass-transfer literature, the nondimensional ratio $L / l_{d}$, where $L$ is a characteristic length scale for the problem, is known as the "Sherwood number". ${ }^{12,13}$ ) Note that Eq. (9) is always valid, since it is based only on the definition of $l_{d}$. The value of $l_{d}$, or, equivalently, the Sherwood number, depends on all properties of the flow (geometry, Peclet number, etc.) and is determined by solving the governing transport equations. If $\mathrm{Pe}<1$ and homogeneous recombination can be neglected, then $l_{d}$ depends primarily on reactor geometry; on the other hand, if $\mathrm{Pe}>1$, then the flow velocity and gas properties also affect $l_{d}$.

Note that from Eq. (8) the quantity $l_{d} / n_{0} D_{\mathrm{H}}$ may be interpreted as a "resistance" for $\mathrm{H}$ transport, with the mole fraction difference as the driving potential. Since $n_{0} D_{\mathrm{H}}$ is independent of pressure and only weakly dependent on temperature, the resistance for $\mathrm{H}$ transport is a function primarily of $l_{d}$. Explicit values for $l_{d}$ are given below.

Let us define another length scale $\lambda_{\mathrm{H}}$ as

$$
\lambda_{\mathrm{H}}=\frac{4 D_{\mathrm{H}}}{\bar{c}_{\mathrm{H}}} .
$$

This length scale is a function only of temperature, pressure, and gas composition, and is independent of flow velocity. Since from simple kinetic theory $D_{\mathrm{H}}$ is roughly given by $\left(\frac{1}{3}\right) \bar{c}_{\mathrm{H}} \lambda_{\mathrm{mfp}}$, ${ }^{13}$ where $\lambda_{\mathrm{mfp}}$ is the mean free path, $\lambda_{\mathrm{H}}$ is approximately equal to the mean free path. In fact, we may take Eq. (10) to define the mean free path for $\mathrm{H}$ transport. Then Eq. (9) may be written as

$$
\frac{X_{\mathrm{H}, 0}}{X_{\mathrm{H}, \mathrm{ref}}}=\left(1+\frac{\gamma_{\mathrm{H}}}{\mathrm{Kn}}\right)^{-1} \text {, }
$$

where 
TABLE I. Diffusion length scales in the low-Pe limit, assuming constant diffusion coefficient and no gas-phase chemistry.

\begin{tabular}{lcc}
\hline \hline Geometry & $l_{d, 1}$ & $l_{d, 2}$ \\
\hline $\begin{array}{l}\text { Infinite parallel } \\
\text { planes separated by } L\end{array}$ & $L$ & $L$ \\
Infinitely long & & \\
concentric cylinders, & & \\
$R_{1}<R_{2}$ & $R_{1} \ln \left(R_{2} / R_{1}\right)$ & $R_{2} \ln \left(R_{2} / R_{1}\right)$ \\
Concentric spheres, & & \\
$R_{1}<R_{2}$ & $R_{1}\left(1-R_{1} / R_{2}\right)$ & $R_{2}\left(R_{2} / R_{1}-1\right)$ \\
\hline \hline
\end{tabular}

$$
\mathrm{Kn}=\frac{\lambda_{\mathrm{H}}}{l_{d}}
$$

is the Knudsen number for $\mathbf{H}$ based on $l_{d}$.

In the limit $\mathrm{Kn}>\gamma_{\mathrm{H}}$, Eq. (11) reduces to $X_{\mathrm{H}, 0}=X_{\mathrm{H}, \mathrm{ref}}$. In this limit, spatial gradients in $\mathrm{H}$ mole fraction are negligible. In the opposite limit $\left(\mathrm{Kn}<\gamma_{\mathrm{H}}\right)$, the $\mathrm{H}$ mole fraction at the surface is much less than that at the reference location. In this limit, gradients in $\mathrm{H}$ mole fraction are large, and Eq. (11) reduces to

$$
\frac{X_{\mathrm{H}, 0}}{X_{\mathrm{H}, \mathrm{ref}}}=\frac{\mathrm{Kn}}{\gamma_{\mathrm{H}}} \text {. }
$$

This limit defines the regime in which $\mathrm{H}$ transport is diffusion limited.

The value of $\lambda_{\mathrm{H}}$ may be estimated for typical CVD diamond growth conditions. For atomic hydrogen diffusing through a bath gas of $\mathrm{H}_{2}, D_{\mathrm{H}}\left(\mathrm{cm}^{2} / \mathrm{s}\right)$ at $1200 \mathrm{~K}$ is approximately $1.7 \times 10^{4} / p,{ }^{8}$ where $p$ is the pressure in Torr. (A few percent hydrocarbon mole fraction does not significantly change $D_{\mathrm{H}}$.) At this temperature, $\bar{c}_{\mathrm{H}}=5 \times 10^{5}$ $\mathrm{cm} / \mathrm{s}$; therefore,

$$
\lambda_{\mathrm{H}} \approx \frac{0.14}{p} \mathrm{~cm},
$$

with $p$ in Torr. Since $\mathrm{H}$ transport begins to become diffusion limited when $\mathrm{Kn} \approx \gamma_{\mathrm{H}}$, if $\gamma_{\mathrm{H}} \approx 0.1$, then at 20 Torr $l_{d}$ must be less than $0.7 \mathrm{~mm}$ to avoid diffusion limitations to $\mathrm{H}$ transport; at $1 \mathrm{~atm}, l_{d}$ would have to be less than $18 \mu \mathrm{m}$. As discussed below, for most current diamond CVD methods $l_{d}$ is not this small; therefore, in most cases $\mathrm{H}$ transport is diffusion limited, and we would expect large spatial gradients in $\mathbf{H}$ concentration within the reactor. In this case, to increase $\mathrm{H}$ at the surface $l_{d}$ should be made smaller; we consider below means to do this both for low-Pe and high-Pe reactors.

\section{DIFFUSION-DOMINATED METHODS}

If $\mathrm{Pe}<1$, then $l_{d}$ is determined by the solution of the diffusion equation. If the diffusion coefficient is constant and homogeneous recombination can be neglected, then $l_{d}$ is a function only of reactor geometry. Some values for simple geometries are given in Table I. Note that for cy- lindrical and spherical geometries $l_{d}$ at the outer surface can be significantly greater than the radius, due to the increase of area with radius.

It is shown in the following section that convectiondominated, high-Pe methods can easily achieve $l_{d}<1 \mathrm{~mm}$. Comparable surface $\mathrm{H}$ concentrations (and therefore growth rates) could in principle be obtained in low-Pe systems if the geometry were such that a similar $l_{d}$ resulted. We show below that in conventional hot-filament and microwave plasma systems $l_{d}$ is of order several centimeters, although by placing the source of atomic hydrogen closer to the substrate, smaller values could be obtained.

\section{A. Hot-filament reactors}

Let us estimate $l_{d}$ and the $\mathrm{H}$ mole fraction at the substrate for a "typical" hot-filament reactor. Quantitative measurements of $\mathrm{H}$ mole fraction in this environment have been made by Schäfer et al. ${ }^{16}$ and Chen et al. ${ }^{17}$ using nonintrusive optical methods, and by $\mathrm{Hsu}^{18,19}$ and Hsu and Tung ${ }^{20}$ using molecular-beam mass spectroscopy, sampling gas through a hole in the substrate. The optical measurements show that the $\mathrm{H}$ concentration decreases with increasing distance from the filament, clearly demonstrating diffusion-limited $\mathrm{H}$ transport away from the filament. ${ }^{16,17}$

In the experiments of $\mathrm{Hsu}$, the conditions employed were a pressure of 20 Torr, an uncoiled filament consisting of a tungsten wire of diameter $0.25 \mathrm{~mm}$ and length $5 \mathrm{~cm}$, and a filament-substrate separation of $1.3 \mathrm{~cm}$. As a first approximation, let us assume that the filament is infinitely long, and that the substrate is an infinitely long cylinder of radius $1.3 \mathrm{~cm}$, concentric with the filament. From Table I, taking $R_{1}=0.0125 \mathrm{~cm}$ and $R_{2}=1.3 \mathrm{~cm}$, we find $l_{d} \approx 6 \mathrm{~cm}$. At 20 Torr, we then find from Eq. (10) that $\lambda_{\mathrm{H}}=70 \mu \mathrm{m}$, and from Eq. (11) we estimate $X_{\mathrm{H}, 0} / X_{\mathrm{H}, \mathrm{fil}} \approx 0.011$, assuming $\gamma_{\mathrm{H}}=0.1$. If it is assumed that the $\mathrm{H}$ mole fraction at the filament corresponds to chemical equilibrium at $T_{\mathrm{fil}}(2600$ $\mathrm{K}$ ), then $X_{\mathrm{H}, \mathrm{fil}}=0.21$. We therefore estimate the value of $X_{\mathrm{H}, 0}$ to be $0.21 \times 0.011=2.4 \times 10^{-3}$. The value measured by $\mathrm{Hsu}$ for these conditions is $2 \times 10^{-3}$ for the lowestmethane case reported $(0.5 \%) .{ }^{18}$ Thus, an estimate based on simple diffusion theory is able to reproduce the experimental result to within experimental uncertainty. (However, an uncertainty of at least a factor of 2 should be assigned to the estimated value, since $X_{\mathrm{H}, \mathrm{fil}}$ is probably somewhat less than the equilibrium value, ${ }^{16}$ and the geometric assumption of an infinitely long filament and a cylindrical substrate will introduce some error.)

To achieve a smaller $l_{d}$ (and therefore a larger $X_{\mathrm{H}, 0}$ ) in a filament system requires moving the filament closer to the substrate or using a larger diameter filament. Taking these ideas to the logical extreme, a conceivable high-rate diamond growth method might be a "sandwich" geometry, in which an electrically heated refractory metal mesh is separated from a water-cooled substrate by a small gap (say 1 $\mathrm{mm}$ ), which contains a hydrogen/hydrocarbon gas mixture. In this case, $l_{d}$ is approximately the gap width, which can be made very small, and film uniformity would not be a problem. While the electrical power requirements would 
be large, they would be no larger than those for current high-rate methods for the same area.

\section{B. Microwave plasma reactors}

Hsu has also measured the $\mathrm{H}$ mole fraction at the substrate in a standard low-Pe microwave plasma system at 20 Torr, and found that the value is $1.2 \times 10^{-3}$-close to that for the filament system. ${ }^{19}$ This suggests that $l_{d}$ for a diffusion-dominated microwave system is also several centimeters. Since we would expect from diffusion theory that $l_{d}$ is of the order of (but somewhat less than) the plasma ball dimensions, this estimate appears reasonable. We would expect, then, that $\mathbf{H}$ transport is usually diffusion limited in low-Pe microwave plasma reactors, in the sense that $X_{\mathrm{H}, 0} \ll X_{\mathrm{H}, \text { plasma }}$.

Rau and Picht ${ }^{21}$ have reported experimental evidence for diffusion-limited film growth in a microwave plasma at 300 Torr, by growing on a substrate containing raised flattened cones. Assuming $l_{d}>1 \mathrm{~cm}$ in this experiment (a conservative estimate), we estimate using the above procedures that $X_{\mathrm{H}, 0} / X_{\mathrm{H} \text {,plasma }}<5 \times 10^{-3}$; therefore $\mathrm{H}$ transport should be strongly diffusion limited. Since $\mathrm{CH}_{3}$ is tightly coupled chemically to $\mathrm{H}$ through the reaction ${ }^{3}$

$$
\mathrm{CH}_{4}+\mathrm{H} \rightleftharpoons \mathrm{CH}_{3}+\mathrm{H}_{2} \text {, }
$$

the $\mathrm{CH}_{3}$ distribution will follow the $\mathrm{H}$ distribution. Since the growth rate under these conditions is expected ${ }^{1}$ to depend on both $[\mathrm{H}]$ and $\left[\mathrm{CH}_{3}\right]$, the spatial dependence of growth rate should reflect the diffusion-limited transport of H. By comparing their experimental results with simulations, Rau and Picht estimate that some species which affects the growth rate has a destruction probability on the surface of 0.1. Although they assume this is the growth species (they do not consider $\mathbf{H}$ transport), this value agrees well with the recombination coefficient of $\mathrm{H}$ on diamond.

These examples have shown that simple diffusion theory is able to rationalize experimental observations in both filament and plasma systems when $\mathrm{Pe}<1$. The $\mathrm{H}$ mole fraction at the substrate can be estimatcd with reasonable accuracy, and the observation of film growth features associated with diffusion-limited transport is explained. Diffusion theory also indicates the modifications needed to achieve higher $\mathrm{H}$ mole fractions at the surface in these systems. Higher input power can be used to fully dissociate hydrogen near the filament or in the plasma; beyond this, it is necessary to decrease $l_{d}$. This is difficult to do in lowpressure, low-Pe plasma systems, since the plasma dimensions are governed by the excitation wavelength. However, modifications of current filament systems could in principle achieve a small $l_{d}$ and become an alternate means of achieving high film growth rates.

\section{CONVECTION-DOMINATED METHODS}

Due to the difficulties of achieving a small diffusion length scale at low $\mathrm{Pe}$, most high-rate diamond growth techniques make use of convective transport. In a high-Pe (or high-Re) flow, the diffusion length scale at the sub-

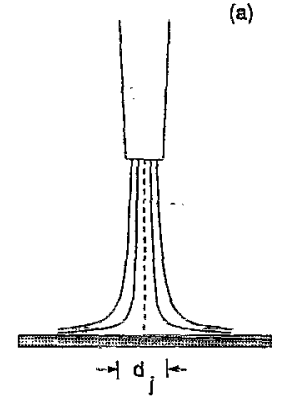

FIG. 1. Typical configurations for high-rate diamond growth: (a) a small high-velocity jet impinging on a larger substrate; (b) a small substrate immersed in a uniform gas flow.

strate is not determined by reactor dimensions, but by an inherent length scale of the flow-the boundary layer thickness.

A typical configuration for high-rate diamond growth is shown in Fig. 1(a). A high-velocity, high-temperature gas jet impinges at normal incidence on a cooled substrate and is deflected radially. A second type of flow, which is used occasionally for diamond growth, ${ }^{6,7,22,23}$ is shown in Fig. 1(b). Here a small substrate is immersed in a larger, uniform gas flow.

The flow in Fig. 1(a) is more difficult to analyze that that in Fig. 1(b), since the effects of viscous drag, heat conduction, and radial species diffusion may significantly broaden and cool the jet after it leaves the torch nozzle before impinging on the substrate. For this reason, we will consider primarily the flow in Fig. 1(b), although most of the results will apply qualitatively to the flow in Fig. 1(a) by substituting $d_{j}$ for $d_{s}$, where $d_{j}$ is an approximate jet diameter at the substrate (or the deposit diameter). We will assume that far from the substrate the flow is uniform and is directed downward with velocity $U_{\infty}$, and has temperature $T_{\infty}$ and species mole fractions $X_{k, \infty}$.

An important flow parameter is the approach Mach number $M_{\infty}$, defined as the ratio of $U_{\infty}$ to the sound speed at $T_{\infty}$. Both subsonic flows $\left(M_{\infty}<1\right)$ and supersonic flows $\left(M_{\infty}>1\right)$ are considered here. We restrict attention to the continuum flow regime, where the mean free path is much less than the substrate dimensions. From Eq. (10), this is reasonable for pressures above a few Torr. (While most convective diamond growth reactors operate in this flow regime, the supersonic arcjet reactor of Loh and Cappelli ${ }^{24,25}$ does not, and therefore is beyond the scope of this analysis.)

We defer the issue of homogeneous chemistry to Sec. IV $\mathrm{B}$, where recombination of $\mathrm{H}$ in the boundary layer is examined, and begin by considering the "frozen flow" limit in which homogeneous chemistry is neglected. The issue of homogeneous chemistry outside the boundary layer (due, for example, to a nonequilibrium freestream, or to the presence of a shock wave) is not considered here.

In high Reynolds number flows, the effects of diffusive transport (viscosity, heat conduction, and molecular species diffusion) are significant only in a thin region near 


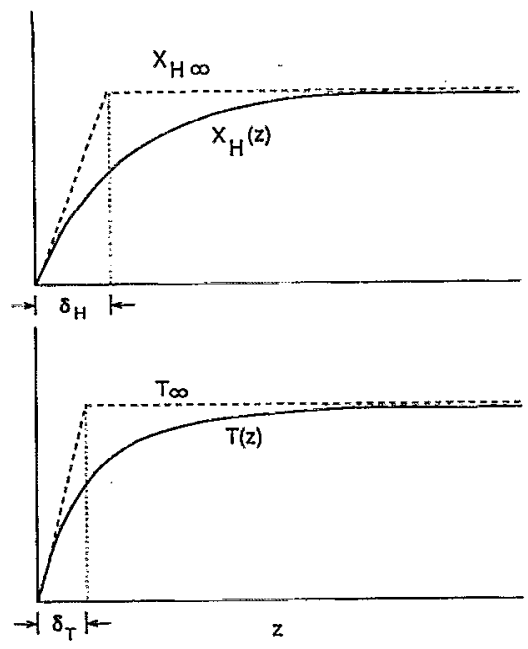

FIG. 2. Geometric interpretation of the boundary-layer conduction thicknesses $\delta_{\mathrm{H}}$ and $\delta_{T}$.

solid surfaces (the boundary layer) and in the wakes behind objects. Within the boundary layer, gradients in velocity, temperature, and species mole fractions are large: The radial velocity rapidly decreases from its value outside the boundary layer (given by potential flow theory) to zero at the surface, and the temperature decreases from $T_{\infty}$ to the substrate temperature. [It may be shown from kinetic theory that the translational gas temperature at the surface must equal the surface temperature if the substrate dimensions are much larger than the gas mean free path. ${ }^{26}$ While temperature discontinuities have been measured ${ }^{27}$ at filaments in hot-filament CVD (HFCVD) reactors, this is possible since the filament diameter is similar to the mean free path.] If a particular species reacts on the surface or in the boundary layer, then its mole fraction will also vary sharply through the boundary layer.

In a gas the boundary layers for velocity, temperature, and species are all similar in thickness, since the transport properties determining each (the kinematic viscosity $v$, the thermal diffusivity $\alpha$, and the diffusion coefficients $D_{k}$, respectively) are all similar in magnitude. The boundary layer thickness may be defined in various ways. For the present purposes, the most useful definition is the conduction thickness. ${ }^{12}$ For $\mathrm{H}$ transport, this is defined as

$$
\delta_{\mathrm{H}}=\frac{X_{\mathrm{H}, \infty}-X_{\mathrm{H}, 0}}{\left(d X_{\mathrm{H}} / d z\right)_{z=0}} .
$$

With this definition for the boundary layer thickness it is clear by comparison with Eq. (7) that the diffusion length scale for $\mathrm{H}$ transport is simply given by $l_{d}=\delta_{\mathrm{H}}$. A similar thermal boundary layer thickness may be defined

$$
\delta_{T}=\frac{T_{\infty}-T_{0}}{(d T / d z)_{z=0}} .
$$

The geometric interpretation of $\delta_{\mathrm{H}}$ and $\delta_{T}$ is shown in Fig. 2.

We are most interested in the boundary layer near the center of the substrate-i.e., near the stagnation stream-
TABLE II. The constant $C$ in Eq. (19) for flow normal to spheres, cylinders, and disks of diameter $D_{s}$ in the limits of low and high approach Mach number (Refs. 26 and 30 ).

\begin{tabular}{lcc}
\hline \hline & \multicolumn{2}{c}{$C$} \\
\cline { 2 - 3 } Substrate geometry & $M_{\infty} \rightarrow 0$ & $M_{\infty} \rightarrow \infty$ \\
\hline Sphere & 3 & 1.14 \\
Cylinder & 4 & 1.14 \\
Disk & $4 / \pi$ & $\sim 0.3$ \\
\hline
\end{tabular}

line. Since the flow decelerates approaching the substrate, the velocity is always small in this region. For this reason, the stagnation-point boundary layer is always laminar, even if the oncoming flow is turbulent. Nevertheless, turbulence may play some role, since it is known that freestream turbulence can enhance heat and mass transfer through the laminar stagnation-point boundary layer. ${ }^{28,29}$ The possible effects of free-stream turbulence will not be considered here.

The boundary-layer thickness at the stagnation point is determined by the transport properties and a single parameter $a$ which characterizes the velocity field outside the boundary layer. The $a$ parameter, known either as the stagnation-point velocity gradient parameter or the strain rate, is defined by

$$
a=\frac{d v}{d r},
$$

where $v$ is the radial (tangential) velocity just outside the boundary layer. For radii not too large, $a$ is independent of $r,{ }^{26}$ and thus the boundary-layer thickness is constant over the central portion of the substrate. It is shown in Sec. IV B that $a^{-1}$ is the characteristic time for species to diffuse through the boundary layer, and therefore $a$ may be thought of also as an inverse boundary-layer diffusion time. From dimensional considerations, the $a$ parameter must scale with the ratio $U_{\infty} / d_{s}$,

$$
a=C \frac{U_{\infty}}{d_{s}} .
$$

The constant $C$ depends on the geometry of the substrate (i.e., disk, sphere, cylinder, etc.), as well as the approach Mach number of the flow $M_{\infty}$. Values for some simple geometries are given in Table II.

If the temperature difference between the free stream and the surface is small, so that the transport properties and density are constant through the boundary layer, then simple analytical expressions for the boundary layer thickness may be obtained. ${ }^{12}$ At the stagnation point,

$$
\delta_{T}=1.32\left(\frac{v}{a}\right)^{1 / 2}\left(\frac{\alpha}{v}\right)^{0.4}
$$

and

$$
\delta_{\mathrm{H}}=1.32\left(\frac{v}{a}\right)^{1 / 2}\left(\frac{D_{\mathrm{H}}}{v}\right)^{0.4},
$$

assuming $\alpha / v$ and $D_{\mathrm{H}} / v$ are not too far from 1.0 
For a dilute hydrocarbon mixture in $\mathrm{H}_{2}$ at $1200 \mathrm{~K}$, $v \approx 8 \times 10^{3} / p \mathrm{~cm}^{2} / \mathrm{s}$, and $\alpha \approx 1.2 \times 10^{4} / p \mathrm{~cm}^{2} / \mathrm{s}$ ( $p$ in Torr) ${ }^{8}$ Therefore,

$$
\delta_{T} \approx \frac{140}{\sqrt{a p}} \mathrm{~cm}
$$

and

$$
\delta_{\mathrm{H}} \approx \frac{160}{\sqrt{a p}} \mathrm{~cm},
$$

with $a$ in $\mathrm{s}^{-1}$ and $p$ in Torr.

Although Eqs. (22) and (23) are derived for the case of constant properties (small temperature changes), comparison with numerical simulation results shows that they are in fact reasonably accurate for typical boundary layers in diamond CVD reactors, with temperature changes of up to $4000 \mathrm{~K}$ through the boundary layer. For the simulations, the gas was assumed to be a $1 \% \mathrm{CH}_{4}$ in $\mathrm{H}_{2}$ mixture in chemical equilibrium outside the boundary layer, and chemistry in the boundary layer was neglected. Both multicomponent mass diffusion and thermal diffusion were included. The boundary-layer thicknesses from the simulations agreed with those predicted by Eqs. (22) and (23) to within $\pm 20 \%$ for free-stream temperatures from 3000 to $5000 \mathrm{~K}$.

Using Eq. (23) for $l_{d}$ in Eq. (11) results in

$$
\frac{X_{\mathrm{H}, 0}}{X_{\mathrm{H}, \infty}}=\left[1+1100 \gamma_{\mathrm{H}}\left(\frac{p \text { (Torr) }}{a\left(\mathrm{~s}^{-1}\right)}\right)^{1 / 2}\right]^{-1} \text {. }
$$

Equation (24) shows that $\mathrm{H}$ transport through the boundary layer is determined by the ratio $p / a$. For optimal $\mathrm{H}$ transport, the flow velocity (or Mach number) should be chosen to give an $a$ value such that the second term in Eq. (24) is of order 1; for smaller $a, \mathrm{H}$ transport will be diffusion limited and therefore $\mathrm{H}$ will be significantly depleted at the substrate, while larger $a$ values do not substantially increase $X_{\mathrm{H}, 0}$. Therefore, since $\gamma_{\mathrm{H}} \approx 0.1, p / a$ should be of order $10^{-4}$ Torr $\mathrm{s}$ or less to avoid significant diffusion limitations to $\mathbf{H}$ transport.

Simulations indicate that typical oxyacetylene torch flames at $1 \mathrm{~atm}$ operate with $a$ in the range $10^{3}-10^{4} \mathrm{~s}^{-1.5}$ Therefore, $p / a \approx 0.08-0.8$ Torr $\mathrm{s}$, which is well into the diffusion-limited regime. On the other hand, some plasma jet reactors may achieve $a$ values as large as $10^{5}-10^{6} \mathrm{~s}^{-1}$ for small substrates (several $\mathrm{mm}$ ). ${ }^{5}$. In this case, $p / a$ is between $2 \times 10^{-4}$ and $2 \times 10^{-3}$ Torr $\mathrm{s}$, near the edge of the diffusion-limited regime. For larger substrates, however, it is likely that here too $\mathrm{H}$ transport is nearly completely diffusion limited.

In the diffusion-limited regime, Eq. (24) reduces to

$$
\frac{X_{\mathrm{H}, 0}}{X_{\mathrm{H}, \infty}}=\frac{9.1 \times 10^{-4}}{\gamma_{\mathrm{H}}}\left(\frac{a\left(\mathrm{~s}^{-1}\right)}{p \text { (Torr) }}\right)^{1 / 2} \text {. }
$$

Since $[\mathrm{H}]_{0}=X_{\mathrm{H}, 0}\left(p / R T_{0}\right)$, for $T_{0}=1200 \mathrm{~K}$,

$$
[\mathrm{H}]_{0}=\frac{1.2 \times 10^{-11}}{\gamma_{\mathrm{H}}} X_{\mathrm{H}, \infty} \sqrt{a p} \mathrm{~mol} / \mathrm{cm}^{3} \text {, }
$$

with $p$ in Torr and $a$ in $\mathrm{s}^{-1}$. This expression shows that if $X_{\mathrm{H}, \infty}$ is constant and boundary-layer chemistry may be neglected, then the $\mathrm{H}$ concentration at the substrate in the diffusion-limited regime is proportional to $\sqrt{a p}$. Therefore, increasing either $a$ or $p$ will result in an increase in $[\mathrm{H}]_{0}$. However, as is discussed in the following sub-section, $a$ cannot be increased indefinitely. Also, at sufficiently high pressures $\mathrm{H}$ will begin recombining homogeneously in the boundary layer, which will set an upper limit on the desirable operating pressure. In the subsections below, we turn to the question of the "optimal" conditions which maximize the $\mathrm{H}$ concentration at the surface. It is shown that there exists both an optimal flow Mach number and an optimal pressure.

\section{A. Optimal Mach number for $\mathbf{H}$ transport}

Since $a=C U_{\infty} / d_{s}$, to increase $a$ for a specified substrate diameter requires increasing the flow velocity $U_{\infty}$. However, there are limits on the velocity to which a gas may be accelerated, since the flow acceleration is provided by expanding the gas through a nozzle. The largest obtainable velocity is of the order of the sonic velocity at the temperature upstream of the nozzle. Therefore, if this temperature is fixed (for example, by materials constraints), then there is a maximum attainable flow velocity, and therefore a maximum $d_{s} a$.

To get an estimate of $a_{\max }$, let us consider a gas with constant specific heat, which is at temperature $T^{0}$ and pressure $p^{0}$ upstream of the nozzle, and is expanded reversibly and adiabatically to a Mach number $M_{\infty}$. (In a real nozzle heat losses and other irreversibilities are significant. In this case, $T^{0}$ and $p^{0}$ should be interpreted as the stagnation temperature and pressure, respectively, downstream of the nozzle, which may be significantly less than the upstream static temperature and pressure.) In this case, ${ }^{31}$

$$
U_{\infty}=M_{\infty}\left(\frac{k R T^{0}}{m}\right)^{1 / 2}\left[1+\left(\frac{k-1}{2}\right) M_{\infty}^{2}\right]^{-1 / 2},
$$

where $k=c_{p} / c_{v}$, and $m$ is the molecular weight of the gas. Note that $U_{\infty}$ asymptotically approaches a finite value as $M_{\infty} \rightarrow \infty$.

Since $a=C\left(M_{\infty}\right) U_{\infty} / d_{s}$, we may substitute from Eq. (27) for $U_{\infty}$ resulting in

$$
a=\frac{1}{d_{s}}\left(\frac{R T^{0}}{m}\right)^{1 / 2} A\left(M_{\infty}, k\right),
$$

where

$$
A\left(M_{\infty}, k\right)=C M_{\infty}\left(\frac{k}{1+[(k-1) / 2] M_{\infty}^{2}}\right)^{1 / 2} .
$$

The function $A\left(M_{\infty}, k\right)$ is plotted in Fig. 3(a) for the two $k$ values 1.3 and 1.7 , which bracket those expected for a mixture of $\mathrm{H}$ and $\mathrm{H}_{2}$. These calculations were done for a spherical substrate, since data are available on the dependence of $C$ on Mach number for this geometry. ( $C$ decreases from 3 at low Mach number to 1.14 at high Mach number. ${ }^{26}$ These data take into account the presence of the bow shock for $\boldsymbol{M}_{m}>1$.) 

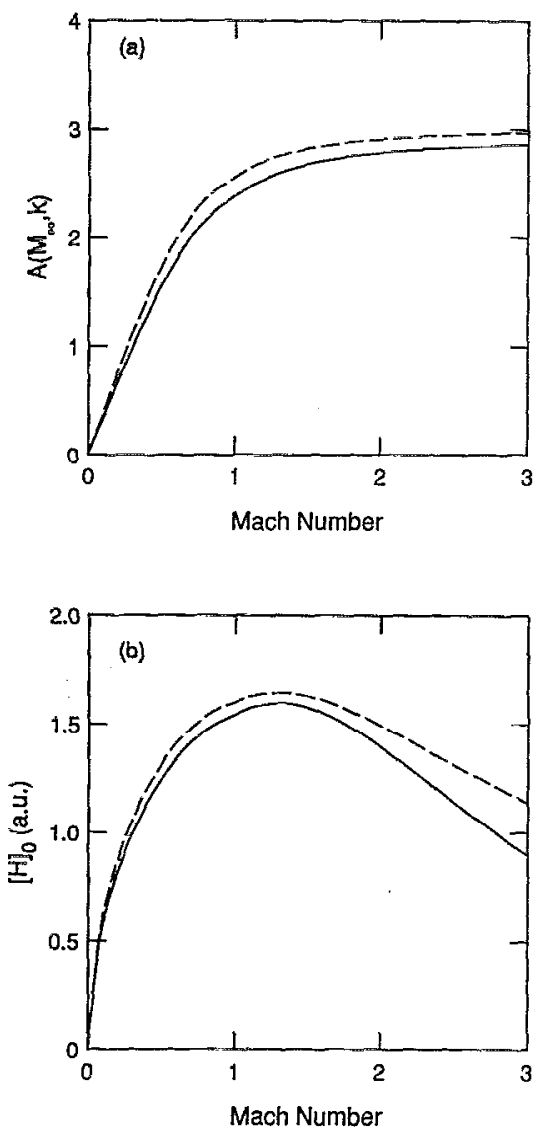

FIG. 3. (a) Dependence of the velocity gradient parameter for flow past a sphere on Mach number; (b) Relative $I$ concentration at the substrate vs Mach number. The solid curves are fur specific heat ratio $k=1.3$, and the dashed curves for $k=1.7$.

The results in Fig. 3(a) show that $A\left(M_{\infty}, k\right)$ attains a limiting value of approximately 3 at high Mach numbers. For a flat disk, the dependence of $C$ on Mach number is not as well known; however, $C$ is estimated to be about a factor of 3 lower than for a sphere at high Mach numbers. ${ }^{26}$ The maximum value of $a$ for a disk is then

$$
a_{\mathrm{max}} \approx \frac{1}{d_{s}}\left(\frac{R T^{0}}{m}\right)^{1 / 2} \text {. }
$$

From this relation it is clear that high $T^{0}$ and low gas molecular weight result in the largest possible velocitygradient parameter. If we take, as extreme values, $T^{0}=10^{4}$ $K$ and $m=1$, then $a_{\max } d_{s} \approx 10^{6} \mathrm{~cm} / \mathrm{s}$. Therefore, if $d_{s}=10$ $\mathrm{cm}$, then $a_{\max } \approx 10^{5} \mathrm{~s}^{-1}$. From Eq. (24), for this substrate diameter $\mathrm{H}$ transport will be necessarily diffusion limited for pressures greater than approximately 10 Torr. Since most high-rate growth methods operate at a pressure significantly higher than this, this calculation illustrates that, in general, large-area growth processes always operate in the diffusion-limited regime.

The effect of molecular weight on $a_{\max }$ is worth noting, since some plasma methods make use of an argon/ hydrogen mixture. If we substitute Eq. (21) into Eq. (9) and use Eq. (30), we find approximately that in the diffusion limit

$$
\left(\frac{X_{\mathrm{H}, 0}}{X_{\mathrm{H}, \infty}}\right)_{\max } \propto \sqrt{D_{\mathrm{H}} a_{\max }}
$$

If the mean molecular weight of the gas is increased by argon addition, both $D_{\mathrm{H}}$ and $a_{\max }$ decrease (since the sound speed is lowered), and therefore the maximum $\mathrm{H}$ concentration achievable at the substrate is lower (in addition to the effects of argon dilution on $X_{\mathrm{H}, \infty}$ ).

In the absence of homogeneous chemistry, the maximum $H$ concentration at the surface will be achieved when the product $a p$ is maximized, where $p$ is the pressure at the substrate. Therefore, the variation of $p$ with Mach number must also be considered. For a subsonic flow, pressure recovery is nearly complete, and therefore $p \approx p^{0}$. For a supersonic flow, however, a shock wave forms above the substrate. Due to the loss of stagnation pressure across the shock wave, $p$ at the substrate will be less than $p^{0}$ if $M_{\infty}>1$. Therefore, for a given upstream stagnation pressure $p^{0}$

$$
[\mathrm{H}]_{0} \propto \sqrt{a\left(p / p^{0}\right)},
$$

where $p / p^{0}$ is the stagnation pressure ratio across the shock. In Fig. 3(b), the dependence of $[\mathrm{H}]_{0}$ on $M_{\infty}$ given by Eq. (32) is shown. For these calculations, $a$ is taken from Fig. 3(a), and $p / p^{0}$ is calculated from standard normal shock relations. ${ }^{31}$ These results show that $[\mathrm{H}]_{0}$ is maximized at a Mach number between 1 and 2 for the frozen flow case (no chemistry).

In reality, the frozen flow assumption may not hold, particularly if a shock wave is present. At the high temperature and pressure behind the shock, the $\mathrm{H}$ mole fraction may equilibrate rapidly; in this case, the value of $X_{\mathrm{H}, \infty}$ (behind the shock but outside the boundary layer) may be dependent on Mach number. Therefore the Mach number at which $[\mathrm{H}]_{0}$ is maximized may differ somewhat from that for the frozen flow case. To better determine the optimal Mach number would require a full reacting flow simulation of the shock layer, which is beyond the scope of the present work.

\section{B. Homogeneous recombination}

In addition to recombination of atomic hydrogen on the diamond surface, $\mathrm{H}$ may recombine in the gas phase at high pressures. This too will affect $H$ at the surface, and will set an upper limit on the pressure at which it is desirable to operate.

To examine the effects of homogeneous recombination, let us consider a flow which, outside the boundary layer, is in chemical equilibrium at $T_{\infty}$. In this case, the $\mathrm{H}$ concentration outside the boundary layer is maintained by the temperature, and recombination only occurs within the boundary layer.

The characteristic time for a $\mathrm{H}$ atom at the edge of the boundary layer to diffuse to the substrate is approximately

$$
\tau_{d} \approx \frac{\delta_{\mathrm{H}}^{2}}{D_{\mathrm{H}}} .
$$

Using Eq. (21) for $\delta_{\mathrm{H}}$ results in 


$$
\tau_{d} \approx a^{-1}
$$

Therefore, the $a$ parameter may be thought of as an inverse boundary-layer diffusion time, in addition to its fluidmechanical interpretation as a velocity gradient.

We may also define an effective first-order recombination rate coefficient $\alpha_{R}$ as

$$
\frac{d[\mathrm{H}]}{d t}=-\alpha_{R}[\mathbf{H}] .
$$

Clearly, homogeneous recombination in the boundary layer will be important whenever $\alpha_{R}>a$. The nondimensional ratio $\alpha_{R} / a$ defines the Damkohler number $\mathrm{Da}_{d}$ for this problem. Recombination is therefore significant when $\mathrm{Da}_{d}>1$.

In a pure hydrogen gas, $\mathbf{H}$ recombines through

$$
\mathrm{H}+\mathrm{H}+\mathrm{H}_{2} \rightarrow 2 \mathrm{H}_{2} \text {. }
$$

In this case,

$$
\alpha_{R}=k_{36}[\mathrm{H}]\left[\mathrm{H}_{2}\right] \text {, }
$$

where $k_{36}$ is the rate constant for this reaction, equal to $9.7 \times 10^{-6} T^{-0.6} \mathrm{~cm}^{6} /\left(\mathrm{mol}^{2} \mathrm{~s}\right) .^{32}$ At constant mole fraction, Eq. (37) shows that $\alpha_{R} \propto p^{2}$. Therefore, for a given degree of dissociation, the condition $\mathrm{Da}_{d}=1$ will be attained when the parameter group $p / a^{1 / 2}$ exceeds some critical value.

With a small amount of added hydrocarbon, a second path competes with reaction (36), and in many cases dominates the recombination rate. This path is due to the two reactions

$$
\mathrm{CH}_{3}+\mathrm{H}+\mathrm{M} \rightarrow \mathrm{CH}_{4}+\mathrm{M}
$$

and

$$
\mathrm{CH}_{4}+\mathrm{H} \rightleftharpoons \mathrm{CH}_{3}+\mathrm{H}_{2} \text {. }
$$

The rate-limiting step is reaction (38), which is in the low-pressure (termolecular) limit even for pressures greater than $1 \mathrm{~atm},{ }^{33}$ and is effectively irreversible. As soon as a methyl is consumed by reaction (38), a $\mathrm{H}$ atom is abstracted from the resulting methane molecule by reaction (39), maintaining a steady-state methyl concentration, but resulting in a net loss of $\mathrm{H}$ atoms. This path is fast, since reaction (38) has no activation barrier ${ }^{33}$ and the barrier for reaction (39) is less than $9 \mathrm{kcal} / \mathrm{mol}^{32}$

Since the $\mathrm{CH}_{3}$ and $\mathrm{CH}_{4}$ concentrations in the boundary layer are strongly affected by boundary-layer chemistry, it is difficult to develop criteria for the significance of homogeneous $\mathbf{H}$ recombination based on simple arguments when hydrocarbons are present. In this case, it is preferable to examine the results of numerical simulation of the boundary layer.

As an example, calculated species profiles are shown in Fig. 4 for a set of conditions where $H$ recombination is significant. For these conditions, acetylene is the most abundant hydrocarbon, and is hardly affected by boundary-layer chemistry. Methane, on the other hand, increases by two orders of magnitude within a few $\mathrm{mm}$ of the substrate. The $\mathbf{H}$ profile is seen to be strongly affected by boundary-layer chemistry.

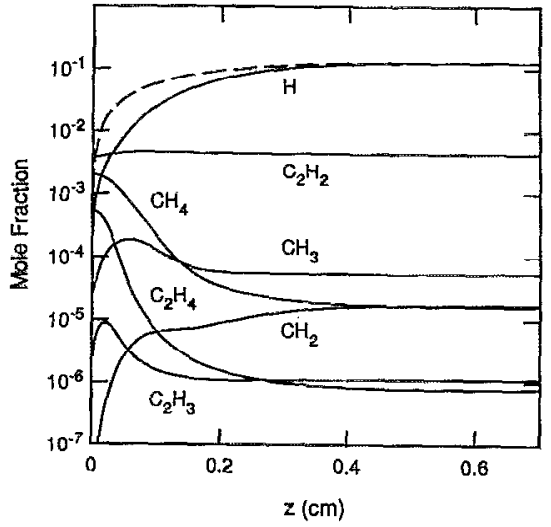

FIG. 4. Selected species profiles calculated for a. $1 \%$ methane/hydrogen mixture, assuming chemical equilibrium outside the boundary layer, with parameter values $T_{\infty}=3000 \mathrm{~K}, a=1000 \mathrm{~s}^{-1}, p=1000$ Torr, $T_{0}=1200 \mathrm{~K}$, and $\gamma_{\mathrm{H}}=0.1$. The dashed line shows the $\mathrm{H}$ profile resulting if boundary layer chemistry is neglected.

The contributions of several reactions to the homogeneous recombination rate for the conditions of Fig. 4 are shown in Fig. 5. Reaction (36) contributes $24 \%$ to the total recombination rate integrated through the boundary layer for these conditions, and reactions (38) and (39) each contribute $34 \%$. Therefore, these three reactions together account for $92 \%$ of the total homogeneous recombination rate. Although acetylene is the most abundant hydrocarbon, the reaction

$$
\mathrm{H}+\mathrm{C}_{2} \mathrm{H}_{2}+\mathrm{M} \rightarrow \mathrm{C}_{2} \mathrm{H}_{3}+\mathbf{M}
$$

only contributes $3 \%$ to the $H$ recombination rate under these conditions.

To quantify the effect of homogeneous recombination, a correction factor $F_{R}$ may be defined as

$$
F_{R}=\frac{X_{\mathrm{H}, 0}}{\left(X_{\mathrm{H}, 0}\right)^{n c}},
$$

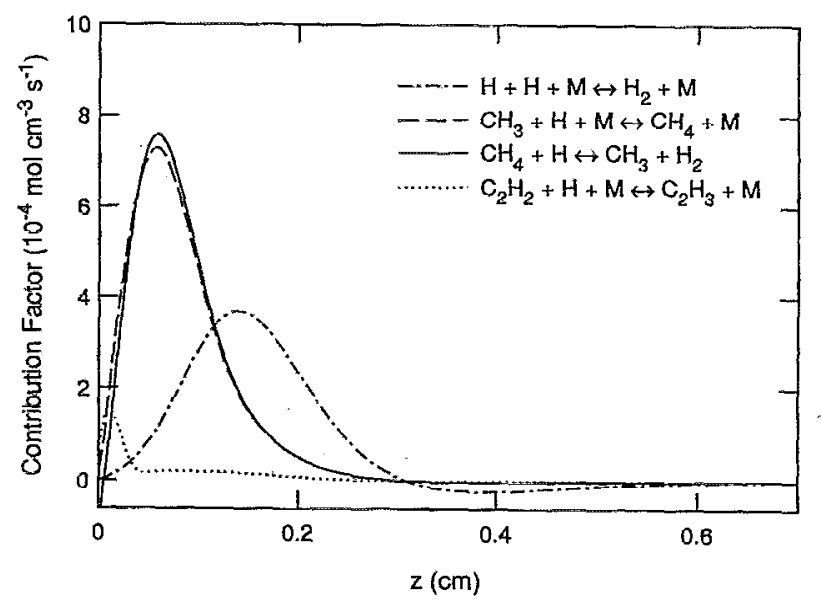

FIG. 5. Contribution factors for several reactions to $\mathrm{H}$ recombination, for the conditions of Fig. 4. 


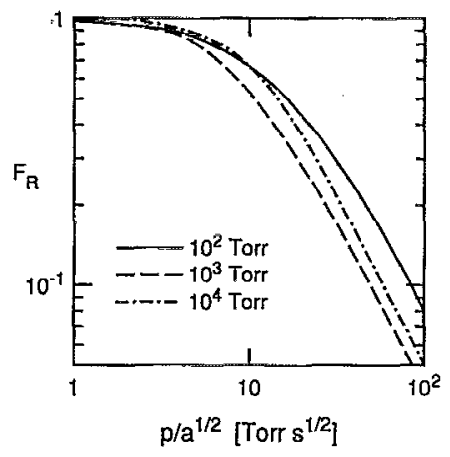

FIG. 6. Ratio of $\mathrm{H}$ mole fraction at the substrate including boundarylayer chemistry to that calculated neglecting boundary layer chemistry vs $p / a^{1 / 2}$. The conditions other than $p$ and $a$ are the same as those for Fig. 4 .

where $\left(X_{\mathrm{H}, 0}\right)^{n c}$ is the $\mathrm{H}$ mole fraction at the substrate neglecting boundary layer chemistry (but still including heterogeneous recombination on the substrate). The $\mathrm{H}$ concentration at the substrate is then determined by multiplying Eq. (26) by $F_{R}$.

Calculations of boundary-layer profiles were carried out with and without boundary-layer chemistry for a range of $a$ and $p$ values, and the correction factor $F_{R}$ determined. The gas was taken to be a mixture of $1 \% \mathrm{CH}_{4}$ in $\mathrm{H}_{2}$, and the gas outside the boundary layer was assumed to be in chemical equilibrium at $T_{\infty}=3000 \mathrm{~K}$. The factor $F_{R}$ is shown in Fig. 6 plotted against $p / a^{1 / 2}$ for the three pressures 100,1000 , and 10000 Torr. It is seen that the dependence of $F_{R}$ on $p / a^{1 / 2}$ is approximately the same for all three pressures, and therefore $p / a^{1 / 2}$ is a valid approximate scaling parameter for homogeneous recombination. Homogeneous recombination is seen to become significant for $p / a^{1 / 2}$ greater than a few Torr $\mathrm{s}^{1 / 2}$.

Since $F_{R}$ falls off approximately linearly with pressure in the regime where recombination is significant, while in the absence of recombination $[\mathrm{H}]_{0} \propto p^{1 / 2}$, for a specified $a$ and $X_{\mathrm{H}, \infty}$ there will be some pressure at which $[\mathrm{H}]_{0}$ is maximized, above which it falls off as $p^{-1 / 2}$. If $X_{\mathrm{H}, \infty}$ also varies with pressure (as it may if the gas outside the boundary layer is in chemical equilibrium), then the scaling with $p$ will be modified, but a maximum at a particular pressure is still expected.

This behavior is shown in Fig. 7, in which simulation results for $[\mathrm{H}]_{0}$ are plotted versus $p$ at fixed $a$ and $T_{\infty}$, assuming chemical equilibrium outside the boundary layer. For $T_{\infty}=3000 \mathrm{~K},[\mathrm{H}]_{0}$ is nearly independent of $p$ over a wide range if boundary-layer chemistry is neglected, due to the decline of the equilibrium value of $X_{\mathrm{H}, \infty}$ with increasing $p$. With chemistry included, $[\mathrm{H}]_{0}$ peaks and then decreases at high $p$. The pressure at which $[\mathrm{H}]_{0}$ is maximal is seen to be just where homogeneous recombination begins to become significant, given approximately by $p / a^{1 / 2}=2$ Torr $\mathrm{s}^{1 / 2}$.

For $T_{\infty}=5000 \mathrm{~K}$, the free stream is nearly fully dissociated up to $10^{3}$ Torr, and the pressure dependence is approximately as $p^{1 / 2}$ at low $p$. For this more activated gas, $[\mathrm{H}]_{0}$ is maximized at $p / a^{1 / 2} \approx 5$ Torr $\mathrm{s}^{1 / 2}$. For $a=10^{5} \mathrm{~s}^{-1}$,

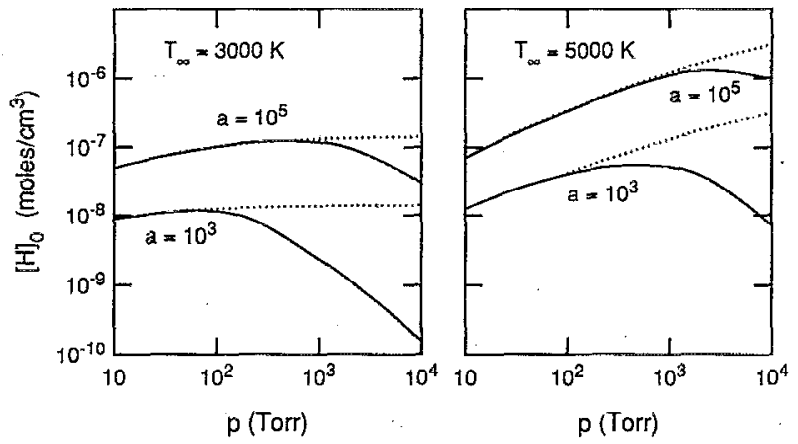

FIG. 7. $H$ concentration at the substrate vs pressure at fixed $a$ and $T_{\infty}$, assuming chemical equilibrium outside the boundary laycr. The dashed curves show the results if boundary-layer chemistry is neglected.

the maximum value of $[\mathrm{H}]_{0}$ is seen to be approximately $10^{-6} \mathrm{~mol} / \mathrm{cm}^{3}$. Since this $a$ value is the largest achievable for a $10-\mathrm{cm}$-diam substrate, and at $5000 \mathrm{~K}$ the free stream is fully dissociated, this $\mathbf{H}$ concentration is an absolute upper bound on the $\mathrm{H}$ concentration achievable by means of convective transport at the surface of a $10-\mathrm{cm}$-diam substrate. The pressure at which this maximum occurs is approximately 2 atm.

\section{Substrate heat flux}

The conditions which lead to high $\mathrm{H}$ concentrations at the surface also result in substantial heat fluxes to the substrate, which may in fact be a limiting consideration for process design. Here we develop an expression for the substrate heat flux for the case where boundary-layer $\mathrm{H}$ recombination can be neglected $\left(p / a^{1 / 2}<1\right.$ Torr $\left.\mathrm{s}^{1 / 2}\right)$.

Substrate heating results primarily from two sources: thermal conduction and heating due to recombination of atomic hydrogen. The heat flux to the substrate may be written as

$$
q_{s}=k\left(\frac{d T}{d z}\right)_{z=0}+\Delta H_{R} \dot{R}_{\mathrm{H}}
$$

where $k$ is the thermal conductivity of the gas at the substrate, $\Delta H_{R}$ is the heat of recombination of atomic hydrogen $(223 \mathrm{~kJ} / \mathrm{mol}$ at $1200 \mathrm{~K})$, and $\dot{R}_{\mathrm{H}}$ is the atomic hydrogen recombination rate (moles of $\mathrm{H} / \mathrm{cm}^{2} / \mathrm{s}$ ). From Eq. $(8)$,

$$
\dot{R}_{\mathrm{H}}=n_{0} \frac{D_{\mathrm{H}}}{\delta_{\mathrm{H}}}\left(X_{\mathrm{H}, \infty}-X_{\mathrm{H}, 0}\right) \text {. }
$$

Using the definition of $\delta_{T}$, Eq. (42) may be rewritten as

$$
q_{s}=\frac{\alpha n_{0}}{\delta_{T}}\left[c_{p} \Delta T+\Delta H_{R}\left(\frac{D_{\mathrm{H}}}{\alpha}\right)\left(\frac{\delta_{T}}{\delta_{\mathrm{H}}}\right) \Delta X_{\mathrm{H}}\right]
$$

where $\Delta T=T_{\infty}-T_{0}$ and $\Delta X_{\mathrm{H}}=X_{\mathrm{H}, \infty}-X_{\mathrm{H}, 0}$. In writing Eq. (44), we have also made use of the relation $k=\alpha n_{0} c_{p}$, where $c_{p}$ is the molar heat capacity at constant pressure at the substrate. Since the ratio $\delta_{T} / \delta_{H}$ is simply $\left(\alpha / D_{H}\right)^{0.4}$ [Eqs. (20) and (21)], we may simplify this to 


$$
q_{s}=\frac{\alpha n_{0}}{\delta_{T}}\left[c_{p} \Delta T+\Delta H_{R}\left(\frac{D_{\mathrm{H}}}{\alpha}\right)^{0.6} \Delta X_{\mathrm{H}}\right] .
$$

Substituting numerical values we have

$$
q_{s}=3.5 \times 10^{-5} \sqrt{a p}\left(\Delta T+8600 \Delta X_{\mathrm{H}}\right),
$$

where $q_{s}$ is in $\mathrm{W} / \mathrm{cm}^{2}, a$ is in $\mathrm{s}^{-1}, p$ is in Torr, and $\Delta T$ is in $K$. The heat flux given by this expression agrees with simulation results to within about $10 \%$. From Eq. (46), it is clear that heat conduction and recombination heating will contribute similar amounts to the total substrate heat flux, since $\Delta T$ may be of order $2000 \mathrm{~K}$, and $\Delta X_{\mathrm{H}}<1$.

Since both the heat flux and the $\mathrm{H}$ concentration at the surface depend on $\sqrt{a p}$, a high $\mathrm{H}$ concentration at the surface will always be accompanied by a large heat flux to the substrate. Some typical values illustrate the magnitudes involved. If we take $a=10^{5} \mathrm{~s}^{-1}, p=1 \mathrm{~atm}$, and $X_{\mathrm{H}, \infty}=0.5$, then $q_{s}=1300 \mathrm{~W} / \mathrm{cm}^{2}$.

For comparison, the heat flux to the substrate during typical oxyacetylene torch growth is roughly $200 \mathrm{~W} / \mathrm{cm}^{2}$, while the heat flux to the water-cooled cathode in an arcjet is of order $2000 \mathrm{~W} / \mathrm{cm}^{2} .{ }^{34}$ For a more extreme case, the heat flux at the throat of the nozzle of the Space Shuttle main engine is of order $13000 \mathrm{~W} / \mathrm{cm}^{2} .{ }^{35}$ In this case, cooling is accomplished using integral cooling passages in the nozzle wall which carry pressurized hydrogen.

These heat fiux estimates show that an important component of reactor design for high growth rates is the substrate thermal design. To handle these heat fluxes and achieve reproducible substrate temperatures, it is critical to eliminate thermal contact resistance and low-conductivity materials. Therefore, metal substrates (for example, molybdenum) with integral cooling passages will be required for high-rate, large-area diamond growth. Since the thermal conductivity of molybdenum at $1200 \mathrm{~K}$ is about 1 $\mathrm{W} / \mathrm{cm} / \mathrm{K},{ }^{36}$ the temperature drop through a $1-\mathrm{mm}$-thick sheet would be $130 \mathrm{~K}$ for a heat flux of $1300 \mathrm{~W} / \mathrm{cm}^{2}$. Therefore, molybdenum would be an acceptable substrate material. Pressurized boiling water would probably be an acceptable coolant. With such a substrate design, heat fluxes of order $1000-2000 \mathrm{~W} / \mathrm{cm}^{2}$ should not present major problems.

\section{PROCESS SCALING RELATIONS}

The results in the previous sections may be combined to yield relations relating growth rate and process efficiency to controllable process parameters. The most important relation for process scaling is Eq. (1), which may be rewritten as

$$
G \propto X_{\text {defl }}[\mathrm{H}]_{0}^{2} .
$$

For a specified defect density (film quality), the sustainable growth rate is predicted to scale quadratically with $[\mathrm{H}]_{0}$. Of course, the model which led to this expression was highly simplified. ${ }^{1}$ For this reason, this quadratic dependence on $[\mathrm{H}]_{0}$ is uncertain. Experimental data on the relation of $G, X_{\text {def }}$ and $[\mathrm{H}]_{0}$ are needed; in the absence of such data, here we explore the scaling implications of Eq. (47).
In the regime where $\mathrm{H}$ transport is diffusion limited but boundary-layer recombination is insignificant, $[\mathrm{H}]_{0}$ $\propto \sqrt{a p} X_{\mathrm{H}, \infty}$ [Eq. (26)]. Using this result in Eq. (47) results in

$$
G \propto(a p) X_{\mathrm{def}} X_{\mathrm{H}, \infty}^{2}
$$

Therefore, to maximize $G$ for a specified $X_{\text {def }}$, the velocity gradient parameter should be maximized (sonic flow), and the pressure at the substrate should be set at that value where homogeneous recombination just begins to become important, as discussed above. Even more important, the gas should be highly activated, since the growth rate for a specified $X_{\text {def }}$ is quadratic in the degree of gas activation $\left(X_{\mathrm{H}, \infty}\right)$.

Note that since $a \propto 1 / d_{s}$, the growth rate at constant defect density is inversely proportional to substrate diameter. Therefore, growing over a larger substrate diameter at the same rate and quality as achieved for small $d_{s}$ requires either increasing the flow velocity (if the flow is subsonic) to maintain the same $a$, increasing the pressure, or achieving higher gas activation.

This also suggests that if it is not necessary to grow a large-area film, then it is preferable to mount several smallarea substrates in the gas flow (such that gas can flow between the substrates) rather than a single substrate of equivalent area. In this case, each small substrate will have a larger $a$ parameter (and therefore thinner boundary layer) than would the single large substrate.

The process efficiency may be more important than the absolute growth rate in many cases. Several different measures of efficiency may be defined-for example, the ratio of mass deposition rate of diamond to the gas flow rate $\eta_{g}$ or the ratio of mass deposition rate to the electrical power required $\eta_{e}$. The mass flow rate per unit area may be expressed in terms of the upstream stagnation temperature $T^{0}$ and pressure $p^{0}$, Mach number $M_{\infty}$, and specific heat ratio $k$. $^{34}$

Assuming that Eq. (48) is valid, and using Eqs. (28) and (29) for $a$, we find that

$$
\eta_{g} \propto \frac{X_{\mathrm{H}, \infty}^{2} T^{0}}{d_{s}}\left(\frac{p}{p^{0}}\right) C\left(M_{\infty}\right)\left[1+\left(\frac{k-1}{2}\right) M_{\infty}^{2}\right]^{1 / k-1},
$$

where, as above, $p / p^{0}$ is the stagnation-pressure ratio across the shock if $M_{\infty}>1$ and is' 1.0 otherwise. This function is shown in Fig. 8, normalized to the value at low Mach number. The efficiency $\eta_{g}$ is higher for supersonic conditions, since although the growth rate is low here, the gas density (and therefore the gas flow rate per unit area) is even lower. However, the increased efficiency (a factor of 2) may not be enough to justify operation under supersonic conditions.

Equation (49) shows that $\eta_{g}$ is strongly dependent on $X_{\mathrm{H}, \infty}$; therefore, the dissociation degree should be maximized for high process efficiency. The process efficiency is independent of pressure, if $\mathrm{H}$ transport is diffusion limited, since both the growth rate and the gas flow rate are linear 


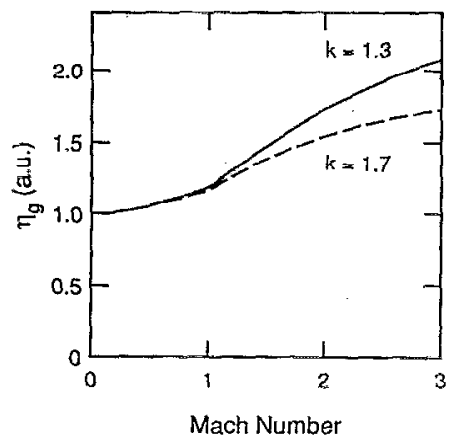

FIG. 8. Predicted scaling of process efficiency $\eta_{g}$ with flow Mach number.

in pressure. The process efficiency is also inversely proportional to $d_{s}$-again, this suggests that there is a price to be paid for large substrate diameter.

Similar scaling relations would hold for $\eta_{e}$. If it is assumed that power consumption is due largely to the energy required to dissociate hydrogen, then $\eta_{e} \propto \eta_{g} / X_{\mathrm{H}, \infty}$. Therefore, the same scaling with pressure, substrate diameter, and Mach number is obtained, but $\eta_{e}$ is now only linearly proportional to $X_{\mathrm{H}, \infty}$.

It should be noted that these conclusions stem from Eq. (47)-in particular, from the result that the growth rate at constant defect density scales faster than linear with $[\mathrm{H}]_{0}$. If, instead, $G$ scaled only linearly with $[\mathrm{H}]_{0}$, then these conclusions would be modified significantly. In particular, in this case the greatest efficiency would be achieved at the lowest pressures. Clearly, experimental data on the scaling of growth rate at constant defect density with $[\mathrm{H}]_{0}$ are needed.

The efficiencies discussed here are "single-pass" efficiencies, which do not account for possible gas or energy cycling. Employing cycling is the most significant way to increase the overall process efficiency. Hydrogen may in principle be indefinitely recycled, and the hydrocarbon feedstock need only be added to make up that lost due to film growth. Also, the heat removed from the substrate and other reactor components (cathodes, walls) can be used to preheat the feedstock gas, or to cogenerate electrical power.

\section{SUMMARY AND CONCLUSIONS}

In this article scaling relations have been developed for atomic hydrogen transport in diamond chemical-vapordeposition reactors. It was shown that simple ideas based on diffusion theory suffice to estimate the $\mathrm{H}$ concentration at the substrate in low-Pe hot-filament and microwave plasma systems. For convection-dominated, high-Pe reactors, $\mathrm{H}$ transport is dominated by the characteristics of the boundary layer. It was shown that most reactors operate in a regime in which $\mathrm{H}$ transport is diffusion limited, in which case the $\mathrm{H}$ concentration at the substrate scales with $\sqrt{a p}$, if homogeneous recombination may be neglected. Loss of $\mathrm{H}$ through homogeneous recombination in the boundary layer was shown to occur primarily through two chemical pathways (direct recombination, and through
$\left.\mathrm{CH}_{3}+\mathrm{H}+\mathrm{M} \rightarrow \mathrm{CH}_{4}+\mathrm{M}\right)$. Since these reactions are termolecular, the effects of homogeneous recombination were shown to depend on $p / a^{1 / 2}$, and to become significant when this parameter exceeds a few Torr $\mathrm{s}^{1 / 2}$.

Finally, the optimal conditions which maximize $\mathbf{H}$ transport were determined for high-Pe reactors. It was shown that approximately sonic flow at a pressure of roughly 1 or $2 \mathrm{~atm}$ is optimal for $\mathrm{H}$ transport, and therefore should allow the highest film growth rates. Achieving these conditions is difficult in current laboratory-scale CVD reactors, due to materials constraints and heat losses. It may be possible to achieve them in large-scale (megawatt) arcjets, or possibly in inductively coupled plasma torches or microwave torches. Also, chemical rockets might provide a similar hot, high-speed gas flow, with additional benefits due to residual oxygen.

There are, of course, many issues not considered here which will affect process scaling and optimization. For example, in plasma reactors lower pressures may be necessary for discharge stability and flow uniformity over reasonable areas. Also, there are economic tradeoffs which must be assessed in comparing different reactor designs to determine conditions which minimize cost. For example, achieving the highest possible growth rate will not lead to the minimum cost if the reactor must be significantly more complex than a simpler design which has a somewhat lower growth rate. Nevertheless, the relations presented here provide a starting point both for optimizing current reactors and designing larger-scale, next-generation reactors.

\section{ACKNOWLEDGMENTS}

Helpful discussions with C. Brennen, A. Acosta, R. Sabersky, N. Glumac, and S. Harris are greatly appreciated. This work is supported, in part, by the National Science Foundation, the Office of Naval Research, and the Naval Research Laboratory.

${ }^{1}$ D. G. Goodwin, J. Appl. Phys. 74, 6888 (1993).

${ }^{2}$ J. E. Butler and R. L. Woodin, Philos. Trans. R. Soc. London 342, 209 (1993).

${ }^{3}$ D. G. Goodwin and G. G. Gavillet, J. Appl. Phys. 68, 6393 (1990).

${ }^{4}$ D. G. Goodwin and G. G. Gavillet, in Proceedings of the 2nd International Conference on New Diamond Science Technology, edited by R. Messier, J. T. Glass, J. R. Butler, and R. Roy (Materials Research Society, Pittsburgh, PA, 1991), pp. 335-340.

${ }^{5}$ D. G. Goodwin, Appl. Phys. Lett. 59, 277 (1991).

${ }^{6}$ T. G. Owano, C. H. Kruger, and D. G. Goodwin (unpublished).

${ }^{7}$ D. S. Green, T. G. Owano, S. Williams, D. G. Goodwin, R. N. Zare, and C. H. Krager, Science 259, 1726 (1993).

${ }^{8}$ R. J. Kee, G. Dixon-Lewis, J. Warnatz, M. E. Coltrin, and J. A. Miller, Technical Repert SAND 86-8246, Sandia National Laboratories, 1986.

${ }^{9}$ J. C. Angus, F. A. Buck, M. Sunkara, T. F. Groth, C. C. Hayman, and R. Gat, MRS Bull. 14, 38 (1989).

${ }^{10}$ T. DebRoy, K. Tankala, W. A. Yarbrough, and R. Messier, J. Appl. Phys. 68, 2424 (1990).

${ }^{11}$ M. A. Kuczmarski, P. A. Washlock, and J. C. Angus, in Applications of Diamond Films and Related Materials, edited by Y. Tzeng, M. Yoshikawa, M. Murakawa, and A. Feldman (Elsevier, Amsterdam, 1991), pp. 591-596.

${ }^{12}$ W. M. Kays and M. E. Crawford, Convective Heat and Mass Transfer, 2nd ed. (McGraw-Hill, New York, 1980).

${ }^{13}$ R. B. Bird, W. E. Stewart, and E. N. Lightfoot, Transport Phenomena (Wiley, New York, 1960). 
${ }^{14}$ S. J. Harris and A. M. Weiner, J. Appl. Phys. 74, 1022 (1993).

${ }^{15}$ L. N. Krasnoperov, I. J. Kalinovski, D. Gutman, and H. N. Chu (unpublished).

${ }^{16}$ L. Schäfer, C. P. Klages, U. Meier, and K. Kohse-Höinghaus, Appl. Phys. Lett. 58, 571 (1991).

${ }^{17}$ K. H. Chen, M. C. Chuang, C. M. Penney, and W. F. Banholzer, J. Appl. Phys. 71, 1485 (1992).

${ }^{18}$ W. L. Hsu, in Proceedings of the 2nd International Symposium on Diamond and Related Materials, edited by A. J. Purdes, K. E. Spear, B. S. Meyerson, M. Yoder, R. Davis, and J. C. Angus (The Electrochemical Society, Pennington, NJ, 1991), pp. 217-223.

${ }^{19}$ W. L. Hsu, J. Appl. Phys. 72, 3102 (1992).

${ }^{20}$ W. L. Hsu and D. M. Tung, Rev. Sci. Instrum. 63, 4138 (1992).

${ }^{21}$ H. Rau and F. Picht, J. Mater. Rcs. 7, 934 (1992).

${ }^{22}$ S. L. Girshick, B. W. Yu, C. Li, and H. Han, Diamond Related Mater. 2, 1090 (1993).

${ }^{23}$ S. L. Girshick, C. Li, B. W. Yu, and H. Han, Plasma Chem. Plasma Proc. 13, 169 (1993).

${ }^{24}$ M. H. Loh and M. A. Cappelli, in Proceedings of the 3rd International Symposium on Diamond Materials, edited by A. J. Purdes, K. E. Spear, B. S. Meyerson, M. Yoder, R. Davis, and J. C. Angus (The Electrochemical Society, Pennington, NJ, in press).
${ }^{25}$ M. H. Loh and M. A. Cappelli, Diamond Related Mater. 2, 454 (1993).

${ }^{26}$ F. M. White, Viscous Fluid Flow (McGraw-Hill, New York, 1974).

${ }^{27}$ U. E. Meier, L. E. Hunziker, D. R. Crosley, and J. B. Jeffries, in Proceedings of the 2nd International Symposium on Diamond and Related Materials, edited by A. J. Purdes, K. E. Spear, B. S. Meyerson, M. Yoder, R. Davis, and J. C. Angus (The Electrochemical Society, Pennington, NJ, 1991), pp. 202-208.

${ }^{28} \mathrm{P}$. K. Macicjewski and R. J. Moffat, J. Heat Transfer 114, 827 (1992).

${ }^{29}$ P. K. Maciejewski and R. J. Moffat, J. Heat Transfer 114, 834 (1992).

${ }^{30}$ H. Lamb, Hydrodynamics, 6th ed. (Dover, New York, 1932), p. 144.

${ }^{31}$ A. H. Shapiro, The Dynamics and Thermodynamics of Compressible Fluid Flow (Wiley, New York, 1953), Vol. 1.

${ }^{32} \mathrm{~J}$. Warnatz, in Combustion Chemistry, edited by W. C. Gardiner, Jr. (Springer, New York, 1984), p. 197.

${ }^{33}$ C. J. Cobos and J. Troe, Z. Phys. Chem. Neue Folge 167, 129 (1990).

${ }^{34}$ P. G. Hill and C. R. Peterson, Mechanics and Thermodynamics of Propulsion (Addison-Wesley, Reading, MA, 1965).

${ }^{35}$ Rocketdyne Div. Rockwell International, "Combustion Devices," Report BC 84-12, 1984.

${ }^{36}$ L. C. Thomas, Heat Transfer (Prentice-Hall, Englewood Cliffs, NJ, 1992). 